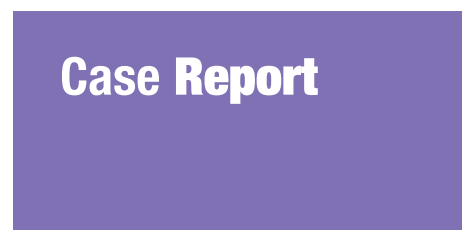

Submitted: 18 Sep 2015

Accepted: 30 May 2016

Online: 29 December 2017

\section{A Rare Presentation of Tubercular Meningitis as Persistent Neutrophilic Meningitis}

\author{
Tarun RALOT ${ }^{1}$, Chander BAFNA ${ }^{1}$, Surender SINGH ${ }^{1}$, Shubhkaran \\ SHARMA ${ }^{2}$ \\ 1 Department of Neurology, RNT Medical College, Udaipur, Rajasthan, India \\ 313001 \\ 2 Gitanjali Medical College \& Hospital, Udaipur, Rajasthan, India 313001
}

To cite this article: Ralot T, Bafna C, Singh S, Sharma S. A rare presentation of tubercular meningitis as persistent neutrophilic meningitis. Malays J Med Sci. 2017;24(6):103-106. https://doi.org/10.21315/mjms2017.24.6.13

To link to this article: https://doi.org/10.21315/mjms2017.24.6.13

\begin{abstract}
Persistent neutrophilic meningitis is an atypical form of chronic meningitis defined as clinical meningitis with a cerebrospinal fluid (CSF) analysis suggestive of neutrophilic pleocytosis that persists for more than one week despite giving empirical antimicrobial therapy. The predominant presentation of tubercular meningitis is with CSF lymphocytic pleocytosis and even if early phase of the disease presents with neutrophilic pleocytosis, no persistence is usually observed for more than one week.

We hereby report the case of an immunocompetent man aged 25 years with tubercular meningitis manifesting as persistent neutrophilic meningitis which is very rare as an entity in tubercular meningitis (TBM). Patient was started on anti-tubercular therapy based on the granulomas in magnetic resonance imaging (MRI) of the brain after which there was a reduction in total cell count in CSF but persisting neutrophilic predominance. Patient improved clinically and was given a full course of anti-tubercular treatment (ATT) thereafter.
\end{abstract}

Keywords: tubercular meningitis, anti-tubercular treatment, persistent neutrophilic meningitis, lymphocytic pleocytosis, granulomas

\section{Introduction}

Persistent neutrophilic meningitis is a variant of chronic meningitis which is vaguely described and is characterised by the neutrophilic persistence in the CSF over prolonged periods of time (more than one week) in association with negative CSF cultures for bacteria and other pathogens with evidence of ongoing meningeal inflammation (2).

There are number of other uncommon infectious and noninfectious causes have been described for persistent neutrophilic meningitis and among them the bacterial pathogens like Streptoccocus pneumoniae, Hemophilus influenzae, Group B streptococci and Neisseria meningitidis account for vast majority of cases $(5,6)$. Other causes include fungal and viral infections like Herpes
Simplex Virus (HSV). The initial management of neutrophilic meningitis usually consists of antimicrobial therapy directed against one or more of these common pathogens $(5,6,9)$.

We hereby report a case of meningeal tuberculosis manifesting as persistent neutrophilic meningitis. This is to our knowledge the first case report of such a rare presentation of TBM as persistent neutrophilic meningitis in our region.

\section{Case Presentation}

Mr X, a 25-year-old man, previously a healthy patient presented to us with chief complaints of fever, headache, vomiting and seizures for four days for which he was admitted in a hospital in his native place and diagnosed as bacterial meningitis based on the Computed 
Tomography (CT) scan and CSF findings and was put on vancomycin and ceftriaxone for two weeks. Patient did not show signs of recovery for which patient was referred to our hospital. Patient had right hemiparesis and aphasia when he was brought to our hospital. We did MRI of the brain which showed multiple conglomerate peripheral enhancing lesions in left occipital lobe likey representing inflammatory granulomas (Figure 1 and Figure 2).

CSF showed Total Leucocyte Counts (TLC) of $700 \mu \mathrm{L}$ with $70 \%$ polymorphs and sugar was $28 \mathrm{mg} / \mathrm{dL}$ with elevated protein levels (Table 1). CSF was negative for Acid Fast Bacilli (AFB) and Indian ink staining. Cultures for bacteria including Mycobacterium tuberculosis, fungus were not positive. HSV (Herpes simplex virus) PCR was also negative.

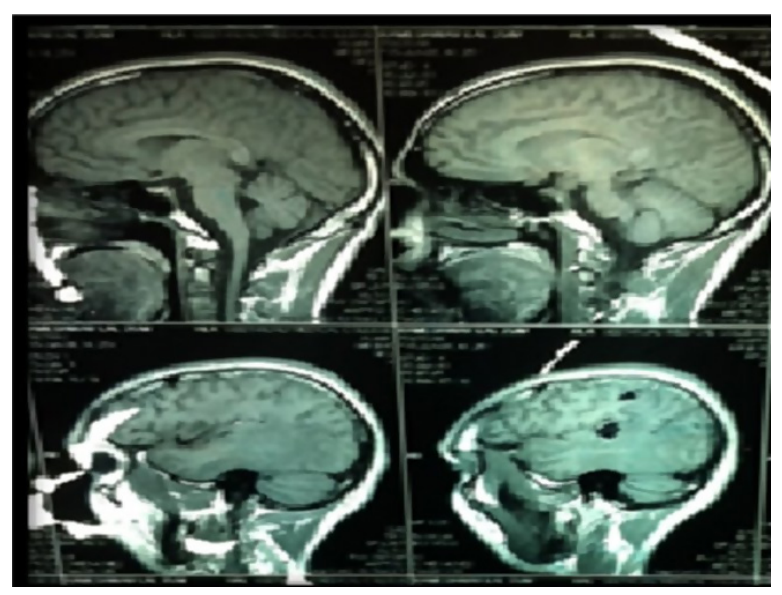

Figure 1. MRI brain TI. Weighted post gadolinium sequence in saggital plane revealed mild leptomeningeal enhancement and ring enhancing lesion in the splenium of corpus callosum
Patient was evaluated with a diagnostic possibility of partially treated meningitis and ATT and anti-epileptic drugs were started in view of the granulomas and seizures. After 10 days of therapy, patient still had low grade fever. Repeat CSF analysis showed reduction in TLC to $400 \mu \mathrm{L}$ with $70 \%$ polymorphs (Table 1 ). After two weeks repeat, the MRI of the brain showed resolution of the granulomas (Figure 3). Repeat CSF at that time showed further reduction in cell counts to $240 \mu \mathrm{L}$ with 40\% polymorphs still persisting. Ruling out all other possible diagnostic probabilities with a good clinical response to ATT and also keeping in view the prevalence of tuberculosis in our region, patient was advised to complete a full course of ATT, AED and review. After a year of ATT regimen patient had fully recovered to his previous healthy state.

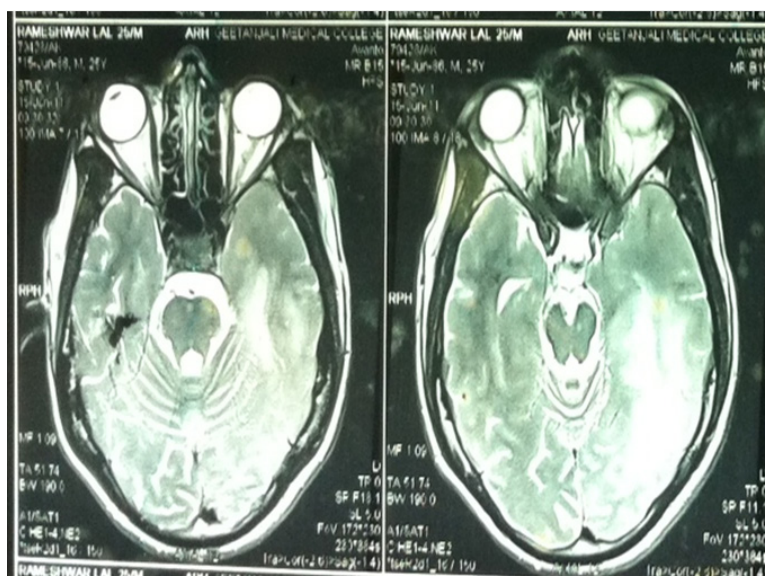

Figure 2. MRI brain T2. Weighted sequence in the axial plane showed hyperintense lesion in the left temporo occipital lobe with marked peri lesional vasogenic edema

Table 1. CSF Analysis (in chronological order)

\begin{tabular}{rcccccc} 
Date & $\begin{array}{c}\text { Cell count } \\
(/ \boldsymbol{\mu L})\end{array}$ & $\begin{array}{c}\text { Polymorphs } \\
(\%)\end{array}$ & $\begin{array}{c}\text { Lymphocytes } \\
(\%)\end{array}$ & $\begin{array}{c}\text { Protein } \\
(\mathbf{m g} / \mathbf{d L})\end{array}$ & $\begin{array}{c}\text { CSF Sugar } \\
(\mathbf{m g} / \mathbf{d L})\end{array}$ & $\begin{array}{c}\text { Blood sugar }^{\mathrm{a}} \\
(\mathbf{m g} / \mathbf{d L})\end{array}$ \\
\hline 2.6 .11 & 3400 & 92 & 8 & 58 & 36 & 85 \\
15.6 .11 & 700 & 70 & 30 & 28 & 95 & 88 \\
27.6 .11 & 400 & 70 & 30 & 29 & 39 & 93 \\
2.7 .11 & 270 & 60 & 40 & 27.7 & 111.7 & 87 \\
2.8 .11 & 200 & 0 & 100 & 27.0 & 100 & 115 \\
\hline
\end{tabular}

${ }^{a}$ Corresponding Blood sugar at the time of CSF analysis 


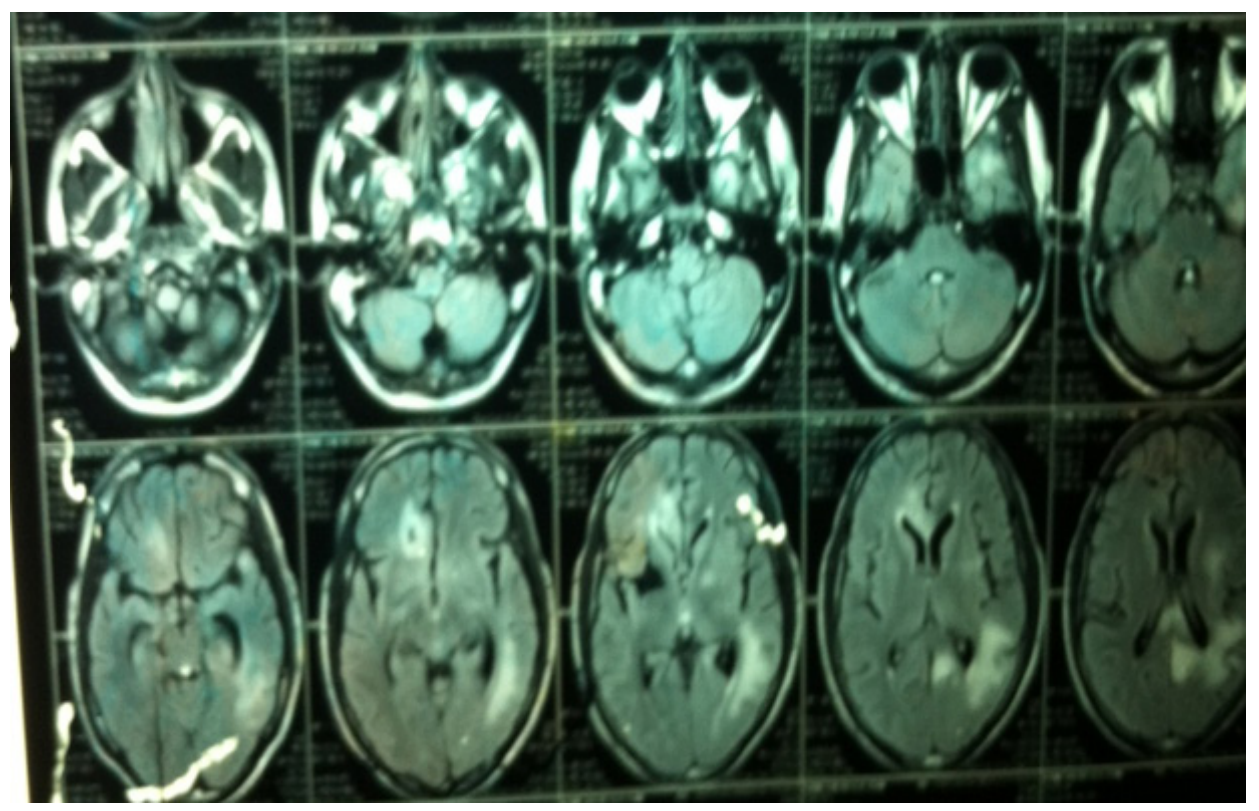

Figure 3. MRI brain FLAIR sequence in the axial plane after two weeks of receiving anti tubercular treatment showed significant reduction in the peri lesional vasogenic edema and ring enhancing lesion in the splenium of corpus callosum is no longer visualised

\section{Discussion}

Tuberculosis is a very rampant disease entity and one of the most common cause of chronic morbidity as far as Indian health conditions are taken into account. This statement is evident from the very fact that India accounts for a TB burden which is highest in the world (21\%) accounting for an incidence of almost 1.9 million cases annually as compared to a global incidence of 9.23 million having a substantial socioeconomic impact (3).

Meningeal tuberculosis stands out as the most common form of central nervous system tuberculosis (TB) and has very high morbidity and mortality. The symptoms of this subacute disease may persist for weeks before it is diagnosed. CSF analysis typical of TBM include a pleocytosis which is lymphocytic-predominant, high protein, and decreased glucose (4-6). Nucleic acid amplification of the CSF by PCR is highly specific but suboptimal sensitivity precludes ruling out TBM with a negative test. CSF AFB smear and culture have relatively low sensitivity but yield is increased with multiple, large volume samples. Treatment for TBM should be initiated as soon as clinical suspicion is supported by initial CSF studies Initial CSF evaluation along with early clinical suspicion warrant treatment initiation (4).
However, neutrophilic pleocytosis is frequently seen, especially in the early phases of disease $(5,6,9)$. If serial CSF analysis is undertaken in patients with early neutrophilic tuberculous meningitis, conversion to a lymphocytic pleocytosis usually ensues within seven days (9). Still occasional reports have documented the persistence of a neutrophilic pleocytosis for longer than one to two weeks $(4,10)$. In fact, some authors have emphasised that marked variability in the CSF cell count with transient increases in CSF neutrophils during the early stages of disease should suggest the diagnosis of tuberculous meningitis (6-8).

Our patient presented with clinical features highly suggestive of meningitis which was labelled to be of tubercular etiology with a strong suspicion keeping in view the appearance of inflammatory granulomas in radioimaging studies and prevalence of disease in our country.

A lack of improvement in CSF parameters suggests either the therapy has been inadequate (suboptimal drug activity, dosage or CSF penetration; resistant organism) or a neurologic complication has developed $(5,6)$. If this lack of improvement in CSF parameters manifests as a persistent neutrophilic CSF profile, especially if bacterial cultures of CSF remain negative, then the clinician is faced with an uncommon and frequently puzzling clinical problem. 
In our patient all the usual and unusual causes of persistent neutrophilic meningitis were effectively ruled out by running all possible tests comprising bacterial, fungal cultures and viral markers which did not show any significant results. Keeping in mind the presence of inflammatory granulomas and endemicity of tuberculosis in our region, we diagnosed and treated him as TBM.

\section{Conclusion}

To conclude, neutrophilic pleocytosis in CSF can be seen in TBM even beyond one month in rare circumstances and the patient should be treated as early as possible on the basis of clinical and radiological findings.further clinical research is still required to give a deeper insight to such rare finding.

\section{Authors' Contributions}

Analysis and interpretation of the data: SHS

Drafting of the article: $\mathrm{CB}, \mathrm{SS}$

Critical revision of the article for important intellectual content: TKR

Final approval of the article: TKR

\section{Correspondence}

Dr Tarun Kumar Ralot

Associate Professor Neurology

MD (Internal Medicine), DM Neurology

(Rajasthan University of Health Science, Jaipur)

Department of Neurology,

RNT Medical College, Udaipur,

Rajasthan, India 313001.

H/P: +919414471653

E-mail: drtarun98@gmail.com

\section{References}

1. Peacock JE Jr. Persistent neutrophilic meningitis. Inf Dis Clinic North America. 1990;4(4):747767 .

2. Peacock JE Jr, McGinnis MR, Cohen MS. Persistent neutrophilic meningitis. Report of four cases and review of the literature. 1984;63(6):379-395.

3. World Health Organization (WHO). Global tuberculosis control; surveillance, planning and financing. WHO Report; 2007.
4. Marx GE, Chan ED. Tuberculous meningitis: diagnosis and treatment overview. Tuberculosis Research and Treatment. 2011;2011:1-9. http:// dx.doi.org/10.1155/2011/798764

5. Hyslop NE Jr, Swartz MN. Bacterial meningitis. Postgrad Med. 1975;58(3):120-128.

6. Swartz MN. Neurology-VIII Infections. In: Rubenstein E, Federman DD, editors. Scientific American medicine. New York: Scientific American Inc.; 1982. p. 11-27.

7. Ellner JJ. Chronic meningitis. In: Mandell GL, Douglas RG Jr, Bennett JE, editors. Principles and practice of infectious diseases. New York: J Wiley and Sons; 1979. p. 761.

8. Ellner JJ, Bennett JE. Chronic meningitis. Medicine. 1976;55:341.

9. McGee ZA, Kaiser AB. Acute meningitis. In: Mandell GL, Douglas RG Jr, Bennett JE. editors. Principles and practice of infectious diseases. New York: Wiley and Sons; 1979. p. 738-76o.

10. Akkara Veetil BM, Yee AH, Warrington KJ, Aksamit AJ Jr, Mason TG. Aseptic meningitis in adult onset Still's disease. Rheumatol Int. 2012;32(12):4031-4032. https://doi. org/10.1007/s00296-010-1529-8 